\title{
Análisis transcultural de los niveles de actividad física y la intención de ser físicamente activo en población adolescente de Chile y España en función de variables sociodemográficas
}

\author{
Cross-cultural analysis of physical activity levels and the intention \\ to be physically active among adolescence students from Chile and Spain \\ regarding sociodemographic variables
}

\author{
Sheila Rodríguez-Muñoz ${ }^{1}$, Laura O. Gallardo ${ }^{1}$, Alberto Abarca-Sos ${ }^{1}$, Alberto Moreno Doña ${ }^{2}$ \\ 1 Facultad de Ciencias Sociales y Humanas (Teruel), Universidad de Zaragoza. España. \\ 2 Facultad de Medicina. Universidad de Valparaíso, Chile.
}

CORRESPONDENCIA:

Sheila Rodríguez Muñoz

sheila.rodriguez.mu@gmail.com

Recepción: octubre 2019 • Aceptación: septiembre 2020
CÓMO CITAR EL ARTÍCULO:

Rodríguez-Muñoz, S., Gallardo, L. O., Moreno Doña, A., \& AbarcaSos, A. (2021). Análisis transcultural de los niveles de actividad física y la intención de ser físicamente activo en población adolescente de Chile y España en función de variables sociodemográficas. Cultura, Ciencia y Deporte, 16(48), 177-185. http://dx.doi. org/10.12800/ccd.v16i48.1628

\section{Resumen}

El principal objetivo fue determinar si existen diferencias en los niveles de actividad física (NAF) y la intención de ser físicamente activo entre países en función del género, curso y estatus socioeconómico. Participaron 3052 adolescentes (14.58 \pm 1.39 años), 1516 chicos y 1536 chicas. Del total, 734 chilenos y 2318 españoles. Las variables medidas fueron los NAF con el "International Physical Activity Questionnaire- Short Form" (IPAQ-SF), la intención de ser físicamente activo con el "Intention to be Physically Active" y el estatus socioeconómico familiar con el "Family Affluence Scale II" (FASII). Se encontraron diferencias significativas en los NAF y en la intención de ser físicamente activo entre países en función del género $(p<.001)$, curso $(p<.001)$ y estatus socioeconómico $(p<.01)$, siendo más altas en España. Además, la relación de cada una de estas variables con los NAF y la intencionalidad varía según el país. Como la relación de las variables sociodemográficas con los NAF y la intención de ser físicamente activo varía en función del país sería necesario llevar a cabo más estudios transculturales que permitan realizar intervenciones de promoción de actividad física y salud adaptadas a las necesidades de cada lugar.

Palabras clave: Transcultural, actividad física, género, estatus socioeconómico, adolescentes.

\section{Abstract}

The main goal was to analyze the physical activity levels and the intention to be physically active differences between both countries regarding the gender, course and socioeconomic status. The sample was 3,052 adolescents ( $14.58 \pm 1.39$ years), 1,516 males and 1,536 females. From the whole participants, 734 were Chilean and 2,318 Spanish. Physical activity levels were measured using the "International Physical Activity Questionnaire- Short Form" (IPAQ-SF), the intention to be physically active using the "Intention to be Physically Active" scale and the socioeconomic status using the "Family Affluence Scale II" (FASII). There are significant differences in physical activity levels and the intention to be physically active between both countries according to the gender $(p<$ $.001)$, course $(p<.001)$ and socioeconomic status $(p<$ .01). Data were higher in Spain than Chile. Moreover, the relation between these sociodemographic variables with physical activity levels and the intention to be physically active were different regarding the country. As the relation between these sociodemographic variables with physical activity levels and the intention to be physically active is different according to the country, cross-cultural researches are required to develop physical activity and health interventions adapted to people`s needs.

Key words: Cross-cultural, physical activity, gender, socioeconomic status, adolescents. 


\section{Introducción}

La inactividad física es actualmente considerada una causante de más de 35 enfermedades crónicas, entre las que se encuentran la diabetes de tipo II, Alzheimer o el cáncer de colon (Booth et al., 2017). A pesar de ello, su presencia es más que evidente y se ha mantenido a lo largo de las últimas décadas, llegándose a hablar de "pandemia de inactividad física" (Kohl et al., 2012).

Uno de los periodos en los que se produce un mayor descenso de esta práctica de actividad física (AF) es la adolescencia (12-18 años). Un $81 \%$ de la población adolescente mundial es insuficientemente activa (Guthold et al., 2020). Es durante este periodo el momento en el cual se producen en esta población ciertos cambios cognitivos (e.g., en el modo de pensar), psicológicos (e.g., en la construcción de la identidad) y sociales (e.g., nuevas amistades, relación familiar) en los jóvenes (OMS, 2014).

La adquisición de hábitos saludables como la práctica de AF comienza a desarrollarse en edades muy tempranas. Su mantenimiento conlleva su estabilidad moderada o alta a lo largo de toda la vida, desde la adolescencia hasta la edad adulta (Telama et al., 2014). Por tanto, para afianzar su mantenimiento, son esenciales las intervenciones durante la juventud, siendo decisivas para mejorar los comportamientos saludables en el futuro (Sevil et al., 2019). Estos cambios que experimentan los adolescentes han supuesto que muchas investigaciones focalicen sus estudios en la práctica de AF en relación a la intención de ser físicamente activo en el futuro (Ruiz-Pérez et al., 2014).

Cuando se comparan estas actitudes hacia los hábitos saludables en poblaciones de diferentes países o continentes, esta intencionalidad de ser físicamente activo puede variar. En un primer estudio llevado a cabo por Brito (2015) entre población ecuatoriana y española, se mostró una mayor intención en los jóvenes latinoamericanos. Posteriormente, se realizaron otros dos estudios en los que abordaron la misma temática: uno en el que los adolescentes españoles mostraron una mayor intención frente a los argentinos (Álvarez et al., 2017a) y otro en el que adolescentes de Colombia y Ecuador presentaron un mayor índice de intencionalidad que los de España (Álvarez et al., 2017b). Por tanto, no hay conclusiones definitivas debido a esta disparidad en los resultados. Es por ello que en la literatura se insiste en la necesidad de realizar estudios transculturales que permitan conocer en qué medida el factor cultural puede llegar a incidir sobre la intencionalidad futura hacia la práctica de AF, pudiendo así desarrollar propuestas de intervención aplicables a varios contextos culturales (Franco et al., 2017).

Además, no solo la cultura, sino también otros factores sociodemográficos (e.g., género, edad, estatus socioeconómico...) han sido considerados como influyentes a la hora de mostrar una mayor o menor intención de práctica (Centeio et al., 2018). Esto promueve la necesidad de realizar más estudios que permitan descifrar las relaciones que se produzcan.

Cuando se habla de niveles de actividad física (NAF), del mismo modo puede existir cierta variación entre los registrados entre continentes, países o incluso ciudades de un mismo país (Tremblay et al., 2016). Estudios recientes muestran que en los países europeos únicamente el 13,6\% de los adolescentes son activos (McMahon et al., 2017). Resultados similares, aunque algo superiores, se muestran en los países latinoamericanos, en los que el porcentaje aumenta a un $15 \%$ (Aguilar-Farias et al., 2018). En ambos estudios, y teniendo en cuenta todos los países participantes, queda evidenciada la gran diferencia que hay en los NAF en función del género, posicionándose a favor del género masculino. Teniendo en cuenta las recomendaciones diarias de AF que la OMS ha establecido para la población adolescente (i.e., 60 minutos diarios de AF moderada-vigorosa), en Chile únicamente el $20 \%$ los alcanza (Aguilar-Farias et al., 2016), mientras que en España es aproximadamente el 37\%, casi el doble (Mielgo-Ayuso et al., 2016). La edad es otra variable que influencia la práctica de $\mathrm{AF}$, ya que los niveles descienden conforme avanzan los años, por tanto, la población es más activa cuanto más joven es (Barr-Anderson et al., 2017). El medio físico también tiene un impacto positivo sobre la $\mathrm{AF}$ tanto en los niños como en los adultos (Smith et al., 2017). Aquellos países que están desarrollados, como España, tienen más posibilidades para la práctica de actividad física que otros países como Chile, considerado por algunos autores como país en vías de desarrollo (Hernández et al., 2010). Del mismo modo, aquellas personas que habitan en vecindarios de un nivel socioeconómico bajo (i.e., con pocas infraestructuras adecuadas y diseñadas para la práctica) tienen menos probabilidad de alcanzar altos niveles de AF (Sallis et al., 2018). Por tanto, los NAF pueden diferir en función de distintos factores sociodemográficos, los cuales resultan más determinantes de la conducta sedentaria y la inactividad física de los adolescentes que los factores sociales (Lavielle-Sotomayor et al., 2014).

Los principales objetivos del estudio fueron: 1) Determinar si existen diferencias en los niveles de actividad física y la intención de ser físicamente activo entre países (España-Chile) en función de otras variables sociodemográficas como el género, curso y el estatus 
socioeconómico familiar. 2) Conocer en qué medida las variables sociodemográficas género, curso y estatus socioeconómico se relacionan con los niveles de actividad física y la intención de ser físicamente activo en cada uno de los países. Por ello, las hipótesis planteadas para la presente investigación fueron: a) Habrá diferencias entre países en los niveles de actividad física y en la intención de ser físicamente activo, siendo mayores en España además de en el género masculino, en menor curso académico y en mayor estatus socioeconómico. b) En ambos países, las variables que más se relacionen con los NAF y la intencionalidad de ser activo serán el género, el curso y el estatus socioeconómico, en dicho orden.

\section{Método}

\section{Participantes}

En un primer momento se propuso la participación a un total de 3060 adolescentes escolarizados en centros de Chile (Región de Valparaíso) y de España (comunidad de Aragón). De todos ellos, ocho sujetos fueron excluidos por no cumplimentar correctamente los cuestionarios entregados. Por tanto, la muestra final se compuso de un total de 3052 estudiantes adolescentes, 734 de nacionalidad chilena $\left(M_{\text {edad }}=14.74\right.$; $D E=1.47)$ y 2318 de nacionalidad española ( $M_{\text {edad }}=$ 14.53; $D E=1.37)$. La muestra de Chile estaba formada por 336 chicos $\left(M_{\text {edad }}=14.80 ; D E=1.47\right)$ y 398 chicas $\left(M_{\text {edad }}=14.70 ; D E=1.47\right)$ de séptimo y octavo básico y de primero a cuarto medio de cinco centros educativos, dos concertados y tres públicos. La muestra de España estaba formada por 1180 chicos $\left(M_{\text {edad }}=14.53\right.$; $D E=1.38)$ y 1138 chicas $\left(M_{\text {edad }}=14.52 ; D E=1.36\right)$ de primero de la ESO a primero de Bachillerato de 14 centros, tres concertados y 11 públicos.

\section{Instrumentos}

\section{Niveles de actividad física}

Se utilizó el cuestionario "International Physical Activity Questionnaire- Short Form" (IPAQ-SF) (Craig et al., 2003). Este cuestionario fue inicialmente diseñado para adultos de entre 18-65 años, aunque posteriormente fue validado en población adolescente (Rangul et al., 2008), así como su versión en castellano (RománViñas et al., 2013). Nos permite conocer tres características específicas de la actividad física realizada por cada sujeto durante los últimos siete días como son la intensidad (i.e., ligera, moderada o vigorosa), la frecuencia (i.e., días por semana) y la duración (i.e., tiempo por día). Está compuesto por un total de siete preguntas (e.g., Durante los últimos siete días, ¿cuántos días realizó usted actividades físicas vigorosas como levantar objetos pesados, jugar a deportes de manera intensa, correr o ir en bicicleta rápido?), cuyas respuestas hacen referencia a los días de práctica (i.e., de cero a siete) y al tiempo invertido (i.e., en minutos). Con los días y los minutos de práctica de las distintas intensidades este cuestionario nos permitirá obtener la actividad física moderada vigorosa (AFMV) diaria de cada sujeto.

\section{Intención de ser físicamente activo}

Se utilizó el cuestionario "Intention to be Physically Active" (Hein et al., 2004), validado en castellano con el nombre de "Medida de Intencionalidad de ser Físicamente Activo" (MIFA) (Moreno et al., 2007). Su finalidad es evaluar la intención futura que los sujetos tienen de ser físicamente activos al finalizar los estudios en el instituto. Está formada por cinco ítems (e.g., Después de terminar el instituto, quisiera formar parte, o continuar, en un club deportivo de entrenamiento), cada uno de ellos con una opción de respuesta en escala tipo Likert que puntúa de uno a cinco, siendo uno "Totalmente en desacuerdo y cinco "Totalmente de acuerdo". La puntuación final de la escala se obtiene con el cálculo de la media aritmética de las puntuaciones obtenidas en cada uno de los ítems, lo que determina que, a mayor puntuación, mayor intención por parte del sujeto de ser físicamente activo en el futuro. Esta versión en castellano (Moreno et al., 2007) tiene una consistencia interna de .94. En el presente estudio la consistencia interna fue .82 .

\section{Estatus socioeconómico familiar (SES)}

Se evaluó a través del cuestionario internacional "Family Affluence Scale II" (FASII) (Boyce et al., 2006), el cual fue validado por estos mismos autores para poder realizar la comparación del nivel socioeconómico familiar entre distintos países. El FAS II se compone de 4 ítems, cada uno con un número diferente de respuestas $y$, en consecuencia, una puntuación diferente (e.g., ¿Tiene tu familia coche, furgoneta o camión? No [cero]; Sí, uno [uno]; Sí. Dos o más [dos]). La puntuación final del cuestionario, denominada índice FAS, se obtiene con el sumatorio de las puntuaciones de las respuestas de cada pregunta, por lo tanto, se sitúa en una escala de cero a nueve puntos. El cuestionario permite la clasificación de los participantes en tres categorías diferentes (nivel socioeconómico bajo [de cero a dos], medio [de tres a cinco] o alto [de seis a nueve]). 


\section{Procedimiento}

Se llevó a cabo el mismo procedimiento tanto para España como para Chile. En primer lugar, se constituyó el cuestionario definitivo, que incluía los cuestionarios adaptados de todas las variables de estudio. Se contó con un especialista en lingüística, que fue el responsable de su adaptación en Chile.

Se comenzó con un primer contacto con el equipo directivo de los distintos centros educativos que tenían implantada la Educación Segundaria Obligatoria en Chile (Región de Valparaíso) y España (comunidad de Aragón). Tras ser planteado el proyecto, si el principal implicado (i.e., tutor/docente a cargo del grupo) mostraba conformidad, se le entregaba una circular, facilitada por los investigadores, en la que se exponía la finalidad del proyecto, así como los compromisos requeridos por parte de sus alumnos (i.e., cumplimentación de un cuestionario). Además, se requirió el consentimiento por parte del padre/madre/tutor. Por otro lado, se incluía una autorización de consentimiento para la participación, que se entregaba a los alumnos junto con los cuestionarios. En ambos escritos (i.e., tanto en la circular como en los cuestionarios) se recalcaba el anonimato de los datos y la máxima sinceridad, ya que tenían un único uso con fines investigadores.

Se acordó con el responsable de grupo una fecha para el pase de cuestionarios. Ese día, los investigadores, junto con la ayuda de los responsables, pasaron los cuestionarios, exceptuando aquellos centros que por motivos de familiarización alumnado-docente prefirieron hacerlo sin investigador en el aula. En España se llevó a cabo durante los meses de febrero, marzo y abril de 2016, mientras que en Chile se llevó a cabo los meses de abril y mayo del mismo año.

\section{Análisis estadísticos}

En primer lugar y tras la confirmación de normalidad en la distribución de la muestra con la prueba de Kolmogórov-Smirnov, se realizó un análisis multivariante (MANOVA) de los NAF y del MIFA en relación al país y otras variables sociodemográficas como el género, el curso y el estatus socioeconómico, además de estadísticos descriptivos para conocer la media y desviación estándar. Posteriormente, se volvió a realizar el mismo análisis con la prueba post-hoc de Scheffé de comparaciones múltiples para determinar si existen diferencias entre los grupos establecidos en las variables curso y estatus socioeconómico. Además, se realizó una prueba $\mathrm{t}$ para muestras independientes que permitió conocer estas diferencias entre países y en función de las distintas variables sociodemográficas.
En segundo lugar se realizó un análisis de regresión lineal por pasos sucesivos sobre los NAF y la intencionalidad de ser físicamente activo como variables dependientes, y utilizando como variables independientes el género, el curso y el estatus socioeconómico. Estos análisis permitieron evaluar en qué medida estas últimas variables se relacionaban las anteriores según el país.

Todos los análisis se realizaron a través del software estadístico SPSS versión 21.0.

\section{Resultados}

Los resultados obtenidos (Tabla 1) tras el análisis multivariante muestran un efecto significativo en el país $(\lambda$ de Wilks $=0.980, F(1,2221)=22.759, p<.001$, $\left.\eta^{2} p=.020\right)$ y también en el género $(\lambda$ de Wilks $=0.980$, $\left.F(1,2221)=22.337, p<.001, \eta^{2} p=.020\right)$. Sin embargo, no mostraron una interacción significativa entre el país y el género $(\lambda$ de Wilks $=0.999, F(1,2221)=$ $0.665, p>.05, \eta^{2} p=.001$ ). Los principales resultados señalan que los NAF diarios son mayores en España que en Chile si se comparan en función del género. Además, en ambos países son siempre mayores en el género masculino. Del mismo modo, en España se presenta una mayor intencionalidad de ser físicamente incluyendo los dos sexos, obteniendo igualmente una mayor intencionalidad en el género masculino.

Por otro lado, tras el análisis multivariante para conocer las diferencias entre países en relación al curso académico (Tabla 2 ), se continúa encontrando un efecto significativo en el país $(\lambda$ de Wilks $=0.988$, $\left.F(1,2222)=12.870, p<.001, \eta^{2} p=.012\right)$, mientras que en cuanto al curso se refiere, los resultados no muestran un efecto significativo $(\lambda$ de Wilks $=0.992$, $\left.F(5,2222)=1.801, p>.05, \eta^{2} p=.004\right)$, ni tampoco mostraron una interacción significativa entre el país y el curso $(\lambda$ de Wilks $=0.995, F(4,2222)=1.317, p>$ $\left..05, \eta^{2} p=.002\right)$. Los NAF en España muestran un claro descenso conforme avanza el curso, presentando un aumento exponencial en el cambio a bachillerato, siendo estos los mayores niveles registrados. Por otro lado, en Chile no se aprecia ningún aumento o decrecimiento constante de los NAF, sino que varían en función del curso, destacando que los mínimos registrados corresponden a lo que sería primero de bachillerato, en este caso tercero medio. Lo mismo ocurre en España con la intencionalidad de ser físicamente activo, encontrando su mayor media en primer curso de la E.S.O. y a partir de ahí se reduce gradualmente. En este caso, el aumento encontrado en el cambio a bachillerato es mínimo, muy similar al del curso ante- 
Tabla 1. Descriptivos y MANOVA de los niveles de actividad física y la intención de ser físicamente activo en función del país y el género.

\begin{tabular}{|c|c|c|c|c|c|c|}
\hline & & Chicos & Chicas & $\mathbf{F}$ & $p$ & $\eta^{2} p$ \\
\hline \multirow[t]{2}{*}{ NAF } & España & $58.59(56.19)^{c}$ & $44.62(49.65)^{a}$ & 29.272 & $<.001$ & .017 \\
\hline & Chile & $49.33(50.62)^{c}$ & $30.90(30.37)^{\mathrm{a}}$ & 27.188 & $<.001$ & .048 \\
\hline \multirow[t]{2}{*}{ MIFA } & España & $4.13(.83)^{a}$ & $3.99(.87)^{\mathrm{a}}$ & 10.779 & $<.01$ & .006 \\
\hline & Chile & $3.90(.92)^{\mathrm{a}}$ & $3.67(.97)^{\mathrm{a}}$ & 7.539 & $<.01$ & .014 \\
\hline
\end{tabular}

Leyenda: NAF = Niveles de Actividad Física; MIFA = Medida de Intencionalidad de ser Físicamente Activo.

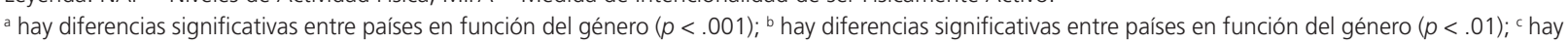
diferencias significativas entre países en función del género $(p<.05)$.

Tabla 2. Descriptivos y MANOVA de los niveles de actividad física y la intención de ser físicamente activo en función del país y el curso-

\begin{tabular}{|c|c|c|c|c|c|c|c|c|c|c|c|}
\hline & & $\begin{array}{c}1^{\circ} \mathrm{ESO} / 7^{\circ} \\
\text { (1) }\end{array}$ & $\begin{array}{c}2^{\circ} \mathrm{ESO} / 8^{\circ} \\
\text { (2) }\end{array}$ & $\begin{array}{c}3^{\circ} \text { ESO/ } \\
1^{\circ} \text { Medio } \\
(3)\end{array}$ & $\begin{array}{c}4^{\circ} \text { ESO/ } \\
2^{\circ} \text { Medio } \\
\text { (4) }\end{array}$ & $\begin{array}{c}1^{\circ} \text { BACH/ } \\
3^{\circ} \text { Medio } \\
\text { (5) }\end{array}$ & $\begin{array}{c}2^{\circ} \text { BACH/ } \\
4^{\circ} \text { Medio } \\
(6)\end{array}$ & $F$ & $p$ & $\eta^{2} p$ & $\begin{array}{c}\text { Orden } \\
\text { del } \\
\text { efecto }\end{array}$ \\
\hline \multirow[t]{2}{*}{ NAF } & España & $\begin{array}{c}58.74 \\
(55.92)^{a}\end{array}$ & $\begin{array}{c}54.68 \\
(56.72)\end{array}$ & $\begin{array}{c}51.52 \\
(55.38)^{c}\end{array}$ & $\begin{array}{c}42.53 \\
(45.12)\end{array}$ & $\begin{array}{c}66.57 \\
(57.25) c\end{array}$ & - & 5.935 & $<.001$ & .014 & $\begin{array}{l}1<4 \\
2<4\end{array}$ \\
\hline & Chile & $\begin{array}{c}37.33 \\
(28.24)^{a}\end{array}$ & $\begin{array}{c}43.53 \\
(61.07)\end{array}$ & $\begin{array}{c}41.80 \\
(37.57) c\end{array}$ & $\begin{array}{c}35.64 \\
(33.74)\end{array}$ & $\begin{array}{c}33.10 \\
(36.28) c\end{array}$ & $\begin{array}{c}35.92 \\
(26.81)\end{array}$ & .831 & $>.05$ & .008 & - \\
\hline \multirow[t]{2}{*}{ MIFA } & España & $\begin{array}{c}4.15 \\
(.80)^{\mathrm{a}}\end{array}$ & $\begin{array}{c}4.12 \\
(.84)^{\mathrm{a}}\end{array}$ & $\begin{array}{c}4.04 \\
(.81)^{\mathrm{a}}\end{array}$ & $\begin{array}{c}3.96 \\
(.99)^{\mathrm{b}}\end{array}$ & $\begin{array}{c}3.98 \\
(1.11)\end{array}$ & - & 3.434 & $<.01$ & .008 & $1<4$ \\
\hline & Chile & $\begin{array}{c}3.88 \\
(.89)^{\mathrm{a}}\end{array}$ & $\begin{array}{c}3.69 \\
(.91)^{\mathrm{a}}\end{array}$ & $\begin{array}{c}3.86 \\
(.93)^{\mathrm{a}}\end{array}$ & $\begin{array}{c}3.76 \\
(.95)^{b}\end{array}$ & $\begin{array}{c}3.49 \\
(1.09)\end{array}$ & $\begin{array}{c}4.10 \\
(1.13)\end{array}$ & 2.040 & $>.05$ & .019 & - \\
\hline
\end{tabular}

Leyenda: NAF = Niveles de Actividad Física; MIFA = Medida de Intencionalidad de ser Físicamente Activo.

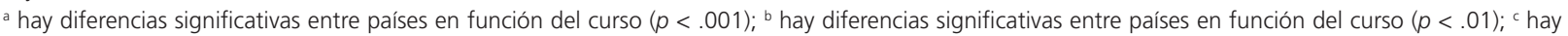
diferencias significativas entre países en función del curso $(p<.05)$.

Tabla 3. Descriptivos y MANOVA de los niveles de actividad física y la intención de ser físicamente activo en función del país y el estatus socio-económico familiar

\begin{tabular}{ccccccccc}
\hline & & Nivel bajo (1) & Nivel medio (2) & Nivel alto (3) & F & p & $\boldsymbol{\eta}^{2} \boldsymbol{p}$ & Orden del efecto \\
\hline \multirow{2}{*}{ NAF } & España & $54.31(52.78)$ & $45.90(50.89)^{\mathrm{a}}$ & $54.25(54.52)^{\mathrm{b}}$ & 4.110 & $<.05$ & .005 & $2<3$ \\
& Chile & $35.28(28.51)$ & $33.19(33.73)^{\mathrm{a}}$ & $44.50(48.45)^{\mathrm{b}}$ & 4.591 & $<.05$ & .017 & $2<3$ \\
\hline \multirow{2}{*}{ MIFA } & España & $3.87(1.18)^{\mathrm{c}}$ & $3.94(.88)^{\mathrm{a}}$ & $4.12(.83)^{\mathrm{a}}$ & 8.092 & $<.001$ & .010 & $2<3$ \\
& Chile & $3.71(.87)^{\mathrm{c}}$ & $3.60(.97)^{\mathrm{a}}$ & $3.91(.93)^{\mathrm{a}}$ & 6.693 & $<.01$ & .025 & $2<3$ \\
\hline
\end{tabular}

Leyenda: NAF = Niveles de Actividad Física; MIFA = Medida de Intencionalidad de ser Físicamente Activo.

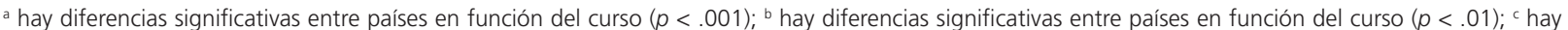
diferencias significativas entre países en función del curso $(p<.05)$.

rior. En Chile vuelve a encontrarse variación en función del curso, encontrando la mayor media en cuarto medio (segundo de bachillerato) seguida de séptimo curso (primero E.S.O.).

Respecto a los NAF en función del estatus socioeconómico familiar (Tabla 3), los resultados del MANOVA vuelven a presentar un efecto significativo en el país $\left(\lambda\right.$ de Wilks $=0.995, F(1,2202)=5.955, p<.01, \eta^{2} p$ $=.005)$ así como en el estatus socioeconómico ( $\lambda$ de Wilks $\left.=0.986, F(2,2202)=7.928, p<.001, \eta^{2} p=.007\right)$. Sin embargo, no se encontró una interacción significativa entre el país y el estatus socioeconómico ( $\lambda$ de Wilks $\left.=0.999, F(2,2202)=.764, p>.05, \eta^{2} p=.001\right)$. Los niveles más bajos en aquellos sujetos que presentan un nivel socioeconómico medio, tanto en España como en Chile. Respecto a la intención de ser física- mente activo, en España aumenta conforme aumenta el nivel socioeconómico, mientras que en Chile sigue siendo más baja en aquellos sujetos con un estatus socioeconómico medio.

Los resultados de análisis de regresión sobre los NAF (Tabla 4) muestran en el modelo final que, en España, tanto el género $(\beta=14.636 ; p<.001)$ como el estatus socioeconómico $(\beta=7.675 ; p=.004)$ se relacionan significativamente y de forma positiva los NAF, siendo el género la que presenta un mayor peso. Sin embargo, el curso se relaciona de forma significativa y negativa $(\beta=-4.760 ; p<.001)$. Por otro lado, el modelo final de Chile excluye la variable curso e incluye únicamente el género $(\beta=17.611 ; p<.001)$ y el estatus socioeconómico ( $\beta=5.930 ; p=.022)$ como variables que se relacionan significativa y positivamente con los 
Tabla 4. Análisis de regresión de los niveles de actividad física diarios por países.

\begin{tabular}{|c|c|c|c|c|c|c|c|c|c|c|c|c|c|c|c|}
\hline & & \multicolumn{8}{|c|}{ España } & \multicolumn{6}{|c|}{ Chile } \\
\hline & & \multirow{2}{*}{ B } & \multirow{2}{*}{ SEB } & \multirow{2}{*}{$\beta$} & \multirow{2}{*}{$p$} & \multirow{2}{*}{$\Delta \mathrm{R} 2$} & \multicolumn{2}{|c|}{$\begin{array}{l}\text { Intervalo de } \\
\text { confianza }\end{array}$} & \multirow{2}{*}{ B } & \multirow{2}{*}{ SEB } & \multirow{2}{*}{$\beta$} & \multirow{2}{*}{$p$} & \multirow{2}{*}{$\Delta \mathrm{R} 2$} & \multicolumn{2}{|c|}{$\begin{array}{c}\text { Intervalo de } \\
\text { confianza }\end{array}$} \\
\hline & & & & & & & $\begin{array}{l}\text { Límite } \\
\text { inferior }\end{array}$ & $\begin{array}{l}\text { Límite } \\
\text { superior }\end{array}$ & & & & & & $\begin{array}{l}\text { Límite } \\
\text { inferior }\end{array}$ & $\begin{array}{l}\text { Límite } \\
\text { superior }\end{array}$ \\
\hline \multirow[t]{3}{*}{ NAF } & Género & 14.64 & 2.57 & .14 & $<.001$ & .005 & 9.60 & 19.67 & 17.61 & 6.27 & .21 & $<.001$ & .009 & 10.66 & 24.56 \\
\hline & Curso & -4.76 & 1.10 & -.10 & $<.001$ & & -6.91 & -2.61 & & & & & & & \\
\hline & SES & 7.68 & 2.63 & .07 & $<.01$ & & 2.52 & 12.83 & 5.93 & 2.57 & .10 & $<.05$ & & .88 & 10.99 \\
\hline \multirow[t]{3}{*}{ MIFA } & Género & .10 & .04 & .06 & $<.01$ & .003 & .03 & .17 & .19 & .07 & .10 & $<.01$ & .010 & .05 & .34 \\
\hline & Curso & -.06 & .02 & -.09 & $<.001$ & & -.09 & -.03 & & & & & & & \\
\hline & SES & .15 & .04 & .09 & $<.001$ & & .08 & .23 & .20 & .05 & .14 & $<.001$ & & .09 & .30 \\
\hline
\end{tabular}

Leyenda: NAF = Niveles de Actividad Física; MIFA = Medida de Intencionalidad de ser Físicamente Activo; SES = Estatus Socioeconómico.

NAF. Igualmente, el género en este país es la variable que más relación tiene con los NAF. El porcentaje de la varianza explicada en el modelo final es de un $0.5 \%$ en España y un $0.9 \%$ en Chile.

Por último, los resultados del análisis de regresión sobre la intencionalidad de ser físicamente activo presentados también en la Tabla 4 muestran en España una relación de la intencionalidad positiva y significativa por parte del género $(\beta=.096 ; p=.007)$ y el estatus socioeconómico $(\beta=.154 ; p<.001)$. Por su parte, el curso académico $(\beta=-.062 ; p<.001)$ se relaciona de nuevo con la intencionalidad (al igual que ocurría con los NAF) de forma significativamente negativa. En Chile nuevamente el modelo final excluye la variable curso, y presenta una relación positiva y significativa con las variables género $(\beta=.194 ; p=.008)$ y estatus socioeconómico $(\beta=.195 ; p<.001)$. En ambos países, el estatus socioeconómico tiene la mayor relación con la intencionalidad de ser físicamente activo. El porcentaje de la varianza explicada en el modelo final es de un $0.3 \%$ en España y un $1 \%$ en Chile.

\section{Discusión}

La primera hipótesis del estudio citaba lo siguiente: "Habrá diferencias entre países en los niveles de actividad física y en la intención de ser físicamente activo, siendo mayores en España además de en el género masculino, en menor curso académico y en mayor estatus socioeconómico". Los principales resultados obtenidos relacionados con esta hipótesis confirman la existencia de diferencias entre países, tanto en los NAF como en la intención de ser físicamente activo, en ambos con mejores resultados en España.

Las diferencias encontradas entre ambos países en relación a los NAF eran predecibles, ya que algunos estudios transculturales anteriores corroboran que estas comparaciones pueden mostrar los distintos patrones que en las diferentes culturas tienen hacia la práctica de AF (Tremblay et al., 2016). Concretamente, se puede hablar de la influencia de la educación hacia la práctica (i.e., las clases de Educación Física), que son diferentes en ambos países, centrándose en una educación mixta en España y una educación segregada por sexos en países latinoamericanos (Álvarez et al., 2017a). Asimismo, el desarrollo de los países ha podido influir en que España presente mayores niveles, ya que tienen más posibilidad y más facilidad de cara a la práctica de actividad física que, en este caso, un país latinoamericano, considerándolos estos últimos por algunos autores como países en vía de desarrollo (Hernández et al., 2010).

El método de medición de los niveles de actividad física también ha podido influir en los resultados, ya que las medidas subjetivas no son tan precisas como las objetivas (Hills et al., 2014). Sin embargo, la medición ha sido la misma para ambos, por lo que se puede entender que las diferencias seguirían existiendo, aunque se utilizara otro método.

El género femenino presenta menores niveles en los dos países, y son todavía menores en las chilenas cuando las comparamos con las españolas. Estos resultados se encuentran en sintonía con otros estudios como el de Aguilar-Farias et al. (2018) y Mayorga-Vega et al. (2019), además, se apoyan en la idea de que el contexto social y cultural son considerados como importantes agentes influyentes sobre las experiencias en la AF de mujeres adolescentes (Yungblut et al., 2012). Asimismo, que los niveles de los chicos chilenos sean también más bajos potencia resultados obtenidos en estudios anteriores con esta misma población (YáñezSilva et al., 2014). Estos autores siguen insistiendo en que, a escala nacional, en Chile hay escasez de estudios que valoren los patrones de AF durante la adolescencia en ambos sexos. 
Pese a que la literatura confirme que la edad está inversamente asociada a los NAF (Barr-Anderson et al., 2017), en este estudio no se puede aceptar para ninguno de los países la hipótesis de que serán los más jóvenes los más activos. Los niveles varían según el país, siendo los de $8^{\circ}$ curso los más activos en Chile y los de $1^{\circ}$ de bachillerato los más activos en España. Los resultados de España se contradicen con las opiniones de algunos autores (Heiss, \& Petosa, 2014), que afirman que a mayor edad mayores responsabilidades, lo que supone la falta de tiempo a la hora de practicar ejercicio físico. Además, estos mismos autores asocian el tener más edad a un mayor desgaste físico, lo que hace que la actividad física sea menos entretenida.

Respecto a los NAF en relación al nivel socioeconómico familiar, en Chile se muestran los mayores NAF en el grupo de población con un nivel socioeconómico alto, mientras que en España en el grupo con un nivel socioeconómico bajo. Una revisión sistemática llevada a cabo por Stalsberg y Pedersen (2010) trató de recopilar todos aquellos estudios que relacionaban la actividad física con el estatus socioeconómico en adolescentes, ya que estos eran mucho menos numerosos que en población adulta. La recopilación de datos no resultó uniforme, lo que puede explicar esta disparidad en los resultados presentes. De los 62 estudios seleccionados, la mayoría de ellos reportaba que los adolescentes con un mayor estatus socioeconómico eran más físicamente activos. Sin embargo, en el $42 \%$ de estos estudios no se reportaba ninguna relación o esta era opuesta, al igual que con los datos obtenidos de España.

En relación a la intención de ser físicamente activo, las diferencias entre países presentadas se contradicen con estudios anteriores como el de Brito (2015) o Álvarez et al. (2017b), en los cuales dicha intención era mayor en aquellos adolescentes latinoamericanos en comparación con la de adolescentes españoles. Sin embargo, se encuentran similitud de resultados en el estudio llevado a cabo por Álvarez et al. (2017a), en el que los adolescentes españoles mostraron una mayor intención frente a los latinoamericanos. En este sentido podemos reiterar la imposibilidad de establecer unas conclusiones definitivas debido a la variedad de resultados. Además, se confirma que la mayor intención se asocia al género masculino, ya que los valores obtenidos para este grupo son mayores tanto en España como Chile (Ruiz-Pérez et al., 2014). Esto podría deberse a que existe una relación positiva significativa entre los NAF y la intención (Sallis et al., 2000), por lo que mayores niveles de AF están relacionados con una mayor intencionalidad de ser físicamente activo.
En ambos países se confirma que la intención de ser activo era mayor en los sujetos de menor curso académico, aunque esta intención no disminuye progresivamente conforme la edad, sino que varía en función del curso. Estos resultados se asemejan a otras investigaciones realizadas (Burns et al., 2014), en las que la mayor intención se reflejaba en el primer curso escolar de la adolescencia, mostrando diferencias significativas con el resto de los cursos. Sin embargo, un estudio realizado por Renner et al. (2007) pone de manifiesto que la $\mathrm{AF}$ es considerada como un estilo de vida en los más jóvenes, mientras que conforme avanza la edad se va convirtiendo en un comportamiento saludable con función de prevención de riesgos. Esta idea se contrapone a los resultados obtenidos, ya que sugiere que esta puede ser la razón por la que la intención de ser físicamente activo es mayor conforme avanza la edad.

Asimismo, los resultados vuelven a coincidir en ambos países cuando se tiene en cuenta el nivel socioeconómico, ya que la mayor intencionalidad de práctica está asociada a un nivel socioeconómico alto. Esta asociación se podría explicar debido a que aquellos con un nivel socioeconómico bajo consideran que tienen insuficientes lugares cercanos a sus viviendas que favorezcan la práctica de actividad física (Kamphuis et al., 2008; Sallis et al., 2018). Por el contrario, aquellas personas con un mayor nivel socioeconómico ven más accesibles los servicios deportivos. Esto les permite asociar esa intención como una posible realidad más fácilmente alcanzable.

La segunda hipótesis del estudio señalaba que "En ambos países, las variables más relacionadas con los NAF y la intencionalidad de ser activo serán el género, el curso y el estatus socioeconómico, en dicho orden". Sin embargo, dicha relación y su orden varía en función del país y de la variable dependiente con la que se relacionen.

Para los NAF en España, las variables sociodemográficas más relacionadas por orden son el género, el estatus socioeconómico y el curso, mientras que en Chile son el género y el estatus socioeconómico, eliminando del modelo de regresión la variable curso. Para la intención de ser físicamente activo, en España encontramos como variable más relacionada el estatus socioeconómico, seguido del género y posteriormente el curso. En Chile vuelve a quedar eliminado el curso y, al igual que en España, la mayor relación se produce con el estatus socioeconómico. Esto puede deberse, como se ha explicado con anterioridad, a que aquellos sujetos con un mayor nivel socioeconómico suelen vivir en espacios con mayores posibilidades para la práctica, por ello ven una mayor facilidad a la hora de comenzar esa práctica, lo que representaría una mayor intención de cara al futuro (Sallis et al., 2018). 
A modo de conclusión, subrayar la importancia de las variables país, género, curso y estatus socioeconómico sobre la $\mathrm{AF}$, ya que se ha corroborado su influencia sobre los NAF de los adolescentes. Además, los resultados fortalecen la idea de seguir realizando estudios transculturales que permitan analizar la relación entre las distintas variables sociodemográficas con la AF y la intención de ser físicamente activos, ya que ejercen un importante efecto sobre estas (LavielleSotomayor et al., 2014) y varían según la localización geográfica. Por tanto, se deberían tener en cuenta para el diseño e implementación de futuros programas de intervención focalizados en población adolescente de distintos continentes, para favorecer así su eficacia en la promoción de hábitos de vida saludable en el futuro (Sevil et al., 2019).

\section{Limitaciones}

En primer lugar, hay que destacar el método de medición subjetiva de los NAF. Pese a que el autoinforme es uno de los instrumentos más utilizados para su medición (Corder et al., 2008), no es tan preciso como pueden ser los instrumentos objetivos, como es el caso de la acelerometría (Hills et al., 2014). Esto es debido a que los datos autorreportados tienden a sobreestimar la realidad (Hagstromer et al., 2010). Por ello, sería conveniente tenerlo en cuenta para futuros estudios. Pese a ello, la medición subjetiva utilizada es científicamente aceptada.
Por otro lado, es reseñable el diseño transversal del estudio, siendo menos completo que aquellos de carácter longitudinal, que permitan conocer y comparar los datos estudiados en diferentes momentos temporales. Asimismo, la temporalización de la toma de datos no se llevó a cabo durante los mismos meses, lo cual puede condicionar notablemente los NAF de los adolescentes.

Por último, a pesar de la amplia muestra participante en el presente estudio, no es una muestra representativa de cada región que nos permita generalizar los resultados obtenidos a toda la población estudiada.

\section{Prospectivas}

Los resultados obtenidos en el presente estudio serían complementados con el diseño e implementación de un programa de intervención basado en aumentar los NAF. Este se diseñaría teniendo en cuenta las variables de estudio, ya que se ha podido confirmar su influencia en los NAF de los adolescentes y es durante la juventud, un momento decisivo para mejorar los comportamientos saludables futuros (Sevil et al., 2019).

\section{Agradecimientos}

A la beca Santander que permitió la financiación de la estancia en Valparaíso y a todos los participantes del estudio, incluyendo instituciones, centros educativos, alumnado, etc.

\section{B I B LIOG R A FÍ A}

Aguilar-Farias, N., Martino-Fuentealba, P., Carcamo-Oyarzun, J., Cortinez-O’Ryan, A., Cristi-Montero, C., Von Oetinger, A., \& Sadarangani, K. P. (2018). A regional vision of physical activity, sedentary behaviour and physical education in adolescents from Latin America and the Caribbean: results from 26 countries. International Journal of Epidemiology, 47(3), 976-986. doi: 10.1093/ije/dyy033

Álvarez, E. F., López, J. C., Gómez, V., \& de Franza, A. L. (2017a). Relación entre motivación, actividad física realizada en el tiempo libre y la intención futura de práctica de actividad física. Estudio comparativo entre adolescentes argentinos y españoles. SPORT TK-Revista EuroAmericana de Ciencias del Deporte, 6(1), 25-34. doi: 10.6018/280371

Álvarez, E. F., López, J. C., Gómez, V., Mesa, J. B., \& Martínez, H. A. (2017b). Influencia de la motivación y del flow disposicional sobre la intención de realizar actividad físico-deportiva en adolescentes de cuatro países. Retos: nuevas tendencias en educación física, deporte y recreación, (31), 46-51.

Barr-Anderson, D. J., Flynn, J. I., Dowda, M., Ross, S. E. T., Schenkelberg, M. A., Reid, L. A., \& Pate, R. R. (2017). The modifying effects of race/ ethnicity and socioeconomic status on the change in physical activity from elementary to middle school. Journal of Adolescent Health, 61(5), 562-570. doi:10.1016/j.jadohealth.2017.05.007

Booth, F. W., Roberts, C. K., Thyfault, J. P., Ruegsegger, G. N., \& Toedebusch, R. G. (2017). Role of inactivity in chronic diseases: evolu- tionary insight and pathophysiological mechanisms. Physiological reviews, 97(4), 1351-1402. doi:10.1152/physrev.00019.2016

Boyce, W., Torsheim, T., Currie, C., \& Zambon, A. (2006). The family affluence scale as a measure of national wealth: validation of an adolescent self-report measure. Social Indicators Research, 78(3), 473-487. doi: 0.1007/s11205-005-1607-6

Brito, J. (2015). Motivación e intención de ser físicamente activo en adolescentes ecuatorianos y españoles. Tesis doctoral. Ciencias.

Burns, C., Murphy, J. J., \& McDonncha, C. (2014). Year in school and physical activity stage of change as discriminators of variation in the physical activity correlate profile of adolescent females. Journal of Physical Activity and Health, 11(4), 721-728. doi: 10.1123/jpah.2011-0353

Centeio, E. E., Cance, J. D., Barcelona, J. M., \& Castelli, D. M. (2018). Relationship between health risk and school attendance among adolescents. American Journal of Health Education, 49(1), 28-32.doi: 10.1080/19325037.2017.1360810

Corder, K., Ekelund, U., Steele, R. M., Wareham, N. J., \& Brage, S. (2008) Assessment of physical activity in youth. Journal of applied physiology. doi:10.1152/japplphysiol.00094.2008

Craig, C. L. et al. (2003). International physical activity questionnaire: 12-country reliability and validity. Medicine and Science in Sports and Exercise, 35(8), 1381-1395. doi: 10.1249/01.MSS.0000078924. 61453.FB 
Ekelund, U., Tomkinson, G., \& Armstrong, N. (2011). What proportions of youth are physically active? Measurement issues, levels and recent time trends. British Journal of Sports Medicine, 45(11), 859-865. doi: 10.1136/bjsports-2011-090190

Franco, E., Coterón, J., Martínez, H. A., \& Brito, J. (2017). Perfiles motivacionales en estudiantes de educación física de tres países y su relación con la actividad física. Suma Psicológica, 24(1), 1-8. doi:10.1016/j. sumpsi.2016.07.001

Guthold, R., Stevens, G. A., Riley, L. M., \& Bull, F. C. (2020). Global trends in insufficient physical activity among adolescents: a pooled analysis of 298 population-based surveys with 1.6 million participants. The Lancet Child \& Adolescent Health, 4(1), 23-35. doi:10.1016/ S2352-4642(19)30323-2

Hagstromer, M., Ainsworth, B. E., Oja, P., y Sjostrom, M. (2010). Comparison of a subjective and an objective measure of physical activity in a population sample. Journal of Physical Activity and Health, 7(4), 541-550. doi: 10.1123/jpah.7.4.541

Hein, V., Müür, M., \& Koka, A. (2004). Intention to be physically active after school graduation and its relationship to three types of intrinsic motivation. European Physical Education Review, 10(1), 5-19. doi: 10.1177/1356336X04040618

Heiss, V., \& Petosa, R. (2014). Correlates of physical activity among adults with type 2 diabetes: a systematic literature review. American Journal of Health Education, 45(5), 278-287. doi: 10.1080/19325037.2014. 933139

Hernández, A., Gómez, L. F., \& Parra, D. C. (2010). Ambientes urbanos y actividad física en adultos mayores: Relevancia del tema para América Latina. Revista de Salud Pública, 12, 327-335.

Hills, A. P., Mokhtar, N., \& Byrne, N. M. (2014). Assessment of physical activity and energy expenditure: an overview of objective measures. Frontiers in nutrition, 1, 5. doi.org/10.3389/fnut.2014.00005

Kamphuis, C. B. M., Van Lenthe, F. J., Giskes, K., Huisman, M., Brug, J., \& Mackenbach, J. P. (2008). Socioeconomic status, environmental and individual factors, and sports participation. Medicine \& Science in Sports \& Exercise, 40(1), 71-81. doi: 10.1249/mss.0b013e318158e467

Kohl, H. W., Craig, C. L., Lambert, E. V., Inoue, S., Alkandari, J. R., Leetongin, G., ..., \& Lancet. (2012). The pandemic of physical inactivity: global action for public health. The Lancet, 380(9838), 294-305.

Lavielle-Sotomayor, P., Pineda-Aquino, V., Jáuregui-Jiménez, O., \& Castillo-Trejo, M. (2014). Actividad física y sedentarismo: Determinantes sociodemográficos, familiares y su impacto en la salud del adolescente. Revista de Salud Pública, 16, 161-172. doi: 10.15446/rsap.v16n2.33329

Mayorga-Vega, D., Saldías, M. P., \& Viciana, J. (2019). Condición física, actividad física, conducta sedentaria y predictores psicológicos en adolescentes chilenos: diferencias por género. Cultura, Ciencia y Deporte, 14(42), 233-241.

McMahon, E. M., Corcoran, P., O’Regan, G., Keeley, H., Cannon, M., Carli, V., ..., \& Balazs, J. (2017). Physical activity in European adolescents and associations with anxiety, depression and well-being. European Child \& Adolescent Psychiatry, 26(1), 111-122. doi: 10.1007/s00787016-0875-9

Mielgo-Ayuso, J., Aparicio-Ugarriza, R., Castillo, A., Ruiz, E., Ávila, J. M., Aranceta-Batrina, J., ... \& González-Gross, M. (2016). Physical activity patterns of the Spanish population are mostly determined by sex and age: findings in the ANIBES study. PloS one, 11(2), e0149969. doi:10.1371/journal.pone.0149969

Moreno, J. A., Moreno, R., \& Cervelló, E. (2007). El autoconcepto físico como predictor de la intención de ser físicamente activo. Psicología y Salud, 17(2), 261-267.
Organización mundial de la salud. Actividad física. 2014. Obtenido de: http://www.who.int/ mediacentre/factsheets/fs385/es/.

Rangul, V., Holmen, T. L., Kurtze, N., Cuypers, K., \& Midthjell, K. (2008). Reliability and validity of two frequently used self-administered physical activity questionnaires in adolescents. BMC Medical Research Methodology, 8(1), 47. doi: 10.1186/1471-2288-8-47

Renner, B., Spivak, Y., Kwon, S., \& Schwarzer, R. (2007). Does age make a difference? Predicting physical activity of South Koreans. Psychology and Aging, 22(3), 482. doi: 10.1037/0882-7974.22.3.482

Ruiz-Pérez, L. M., Ramón-Otero, I., Palomo-Nieto, M., Ruiz-Amengual, A., \& Navia-Manzano, J. A. (2014). La intención de practicar en el futuro en escolares adolescentes. Universidad Europea de Madrid. Kronos, v.13, n.2, 2014.

Sallis, J. F., Conway, T. L., Cain, K. L., Carlson, J. A., Frank, L. D., Kerr, J., ... \& Saelens, B. E. (2018). Neighborhood built environment and socioeconomic status in relation to physical activity, sedentary behavior, and weight status of adolescents. Preventive medicine, 110, 4754. doi:10.1016/j.ypmed.2018.02.009

Sallis, J. F., Prochaska, J. J., \& Taylor, W. C. (2000). A review of correlates of physical activity of children and adolescents. Medicine and Science in Sports and Exercise, 32(5), 963-975. doi: 10.1123/jpah.2011-0353

Sevil, J.; Abós, A.; Sanz-Remacha, M.; Estrada, S. \& Corral, A. (2019). Variability in the compliance with 24-hour movement guidelines between week and weekend days in adolescents of the city of Huesca. ESHPA - Education, Sport, Health and Physical Activity. 3(1): 160-174. doi: http://hdl.handle.net/10481/53935

Smith, M., Hosking, J., Woodward, A., Witten, K., MacMillan, A., Field, A., ... \& Mackie, H. (2017). Systematic literature review of built environment effects on physical activity and active transport-an update and new findings on health equity. International Journal of Behavioral Nutrition and Physical Activity, 14(1), 158. doi:10.1186/s12966-0170613-9

Stalsberg, R., \& Pedersen, A. V. (2010). Effects of socioeconomic status on the physical activity in adolescents: a systematic review of the evidence. Scandinavian Journal of Medicine \& Science in Sports, 20(3), 368383. doi: 10.1111/j.1600-0838.2009.01047.x

Telama, R.; Yang, X.; Leskinen, E.; Kankaanpa"a", A.; Hirvensalo, M.; Tammelin, T.; Viikari, J. S. A., \& Raitakari, O. T. (2014). Tracking of physical activity from early childhood through youth into adulthood. Medicine and Science in Sports and Exercise, 46(5), 955-962. doi:10.1249/MSS.0000000000000181

Tremblay, M. S., Barnes, J. D., González, S. A., Katzmarzyk, P. T., Onywera, V. O., Reilly, J. J., ..., \& Global Matrix 2.0 Research Team. (2016). Global Matrix 2.0: report card grades on the physical activity of children and youth comparing 38 countries. Journal of Physical Activity and Health, 13(11), 343-366. doi: 10.1123/jpah.2016-0594

Viñas, B. R. et al. (2013). Validación en población catalana del cuestionario internacional de actividad física. Gaceta Sanitaria, 27(3), 254-257. doi: 10.1016/j.gaceta.2012.05.013

Yáñez-Silva, A., Hespanhol, J. E., Gómez Campos, R., \& Cossio-Bolaños, M. (2014). Valoración de la actividad física en adolescentes escolares por medio de cuestionario. Revista chilena de nutrición, 41(4), 360366. doi: 10.4067/S0717-75182014000400003

Yungblut, H. E., Schinke, R. J., \& McGannon, K. R. (2012). Views of adolescent female youth on physical activity during early adolescence. Journal of Sports Science \& Medicine, 11(1), 39. 